\title{
Numerical study of heat transfer by natural convection in concentric hexagonal cylinders charged with a nanofluid
}

\author{
TALOUB DJEDID ${ }^{1}$, BOURAS ABDELKRIM ${ }^{2}$, ZIED DRISS $^{3}$ \\ ${ }^{1,2}$ Department of Physics, Faculty of Sciences, University Mohamed Boudiaf of M'sila, ALGERIA \\ ${ }^{1}$ Laboratory of Materials Physics and its Applications, University Mohamed Boudiaf of M'sila, \\ ALGERIA \\ ${ }^{3}$ Department of Mecanics, Electromechanical Systems Laboratory, University of Sfax, TUNISIA
}

\begin{abstract}
In this document, a numerical study of the natural convection of steady-state laminar heat transfer in a horizontal ring between a heated hexagonal inner cylinder and a cold hexagonal outer cylinder. $\mathrm{A} \mathrm{Cu}-\mathrm{water}$ nanofluid traverses this annular space. The system of equations governing the problem was solved numerically by the fluent calculation code based on the finite volume method. Based on the Boussinesq approximation. The interior and exterior sides from the two cylinders are maintained at a fixed temperature. We investigated the impacts of various thermal Rayleigh numbers $\left(10^{3} \leq R a_{t} \leq 2.5 \times 10^{5}\right)$, and the volume fraction from the nanoparticles $(0 \leq \emptyset \leq 0.12)$ on fluid flow and heat transfer performance. It is found that in high thermal Rayleigh numbers, a thin thermal boundary layer is illustrated at the flow that heavily strikes the ceiling and lower from the outer cylinder. In addition, the local and mean Nusselt number from a nanofluid are enhanced by enhancing the volume fraction of the nanoparticles. The results are shown within the figure of isocurrents, isotherms, and mean and local Nusselt numbers. Detailed results of the numerical has been compared with literature ones, and it gives a reliable agreement.
\end{abstract}

Key-Words: - Natural convection, hexagon, thermal Rayleigh numbers, nanofluid, volume fraction.

Received: May 28, 2021. Revised: November 26, 2021. Accepted: December 19, 2021. Published: January 6, 2022.

\section{Introduction}

The natural convection of nanofluids has attracted exceptional understanding from researchers' observation due to its presence in nature and engineering applications such as room ventilation, solar basins, and reactor insulation. The necessity to enhance the thermal transfers of fluids has presented rise to the generation of a new class, called nanofluids. Holding nanoparticles suspended these solutions within a basis fluid. Gratitude to their thermic performance-enhancing properties, nanofluids can be applied in many fields such as the environment, production, cooling of electronic components, biology, medical diagnostics, water treatment, cooling in a heat engine Tzeng et al. [1], and energy storage. However, the main difficulty encountered with nanofluids is to disperse in a stable manner over time the nanoparticles in the base liquid because of their agglomeration, due either to gravity or to temperature-dependent precipitation. While the event of thermic convection in nanofluids, which are usually excellent conductors, both thermic and electrical, and into the attendance of a magnetic domain, two-volume forces control the nanofluid namely: the buoyance force and that of Lorentz. The end can cause magnetohydrodynamics (MHD).

The improvement from heat transfer by convection is the main object of several works, and to do so, a considerable number of investigations have carried out a throng of theoretical, experimental, and numerical essays relating to the representation of the aspects governing convection, the effect of the nature of the systems in which it takes place and the properties of the fluids involved, which are directly related to our study. Leong et al. [2] have studied the flow induced by buoyancy in the porous layer. A parametric study was performed to investigate the effects of Rayleigh number, Darcy number, porous sleeve thickness, and relative thermal conductivity on heat transfer. Zhou et al [3]. Examined the natural convection in a rectangular cavity by the method of Boltzmann. The results show that the Rayleigh number and the solid size fraction of the nanoparticles affect the improvement of the heat transfer of the nanofluids, and there is a critical value of the Rayleigh number on the improved performance of the heat transfer. Al-Asad et al. [4] studied the impact of a rectangular heat source in closed space on the natural convective flow through 
the triangular cavity. Moghari et al. [5] have studied numerically the pressure loss and thermal properties of the flow of nanofluid $\mathrm{Al}_{2} \mathrm{O}_{3}$ water in horizontal rings. Each single-phase fluid method was selected for the modeling of nanofluids. The effects of some important parameters studied and discussed in detail. They have observed that there are significant increases in pressure loss and in elevating energy, which is not acceptable. Izadi et al. [6] examined numerically the influence from the ratio of the Richardson numbers about the walls at the laminar combined convection of a nanofluid circulating within a uniformly heated annulus. The secondary currents and shape of dimensionless axial speed and dimensionless temperature are showed and explained. Seyyedi et al. [7] simulated heat transfer by natural convection under uniform heat flow into an annular enclosure loaded by nanofluids. They used the finite element approach based on the control volume. To evaluate the impact of thermic conductivity and viscidity from the nanofluid they used the Maxwell-Garnetts (MG) and Brinkman models. These results explicate that increasing the aspect report raises the values of the mean Nusselt number. Dawood et al. [8] published a numerical simulation of increasing heat transfer employing nanofluids within an elliptical ring with a regular heat flow boundary state. Four different types of nanofluids of various sizes from nanoparticles were used. They found that glycerin exhibited the best improvement in heat transfer related before the other base fluids were tested. Garoosi et al. [9] performed numerical research at the heat transfer by natural convection from nanofluid inside a rectangular cavity including different pairs from heaters and icehouse. The surfaces from the cavity are protected and many pairs of heating and cooling devices with isothermal surfaces are placed inside the cavity. The influences of many parameters about the heat transfer flow and the dispersion of nanoparticles are investigated. These results designate that the location of the coolers has several important impacts on the heat transfer rate. They noticed that there is an optimum volume fraction of nanoparticles by any thermal Rayleigh number into which the best heat transfer flow can be achieved. Mehrizi et al. [10] applied Boltzmann's approach to exploring the influence of nanoparticles on heat transfer by natural convection in a ring-shaped enclosure, which formed within a heated triangular internal surface and a circular external surface. The impact of the volume fraction from nanoparticles on improving heat transfer has been tested at various thermal Rayleigh numbers. In addition, the influence concerning diagonal, horizontal, and vertical eccentricities in different situations is tested at $\mathrm{Ra}=10^{4}$. These effects prove that the Nusselt number and the best flux rise with rising the fixed volume fraction. The mean Nusselt number rises as the internal cylinder goes descending, but reduces as the position of the internal cylinder moves horizontally. Arbaban et al. [11] improved the heat transfer into horizontal concentric rings with eight radial flippers connected to the internal cylinder using nanofluids. These results confirm that the mean Nusselt number rises with the rising volume fraction and thermic conductivity from nanoparticles. In addition, it is mentioned that the mean Nusselt number from $\mathrm{Cu}$-water nanofluid is the most important with the nanofluids. Boulahia et al. [12] numerically examined the performance from natural and combined convection from $\mathrm{Cu}$-water nanofluid in square forms including circular warming and cooling cylinders inside. The effects of the position and size from circular shapes at the flow of heat transfer are studied. The results confirm that the optimal heat transfer is achieved by locating the circular form near the floor wall, and the rate concerning heat transfer rises with the change in guidance from the pair of circular shapes from the horizontal directorate to the vertical directorate. Hamid et al. [13] studied the increased warmth transfer and hydromagnetic flux of water-founded carbon nanotubes in a partly heated square finformed cavity. Inside the cavity, a fine heated bar is fixed as a heat source. In the calculation, the numerical finite element approach is performed utilizing the Galerkin method. These results illustrated that local Nusselt numbers are enhanced with injecting both a water-based solid carbon volume fraction and radiation influences, while the Nusselt number is maximal in the angles. Lakshmi et al. [14] analytically investigated the natural convection from water-copper nanoliquids into saturated cylindric porous rings. The internal and external perpendicular radial surfaces are subjected to regular mass and heat fluxes and outflows, respectively. The thermophysical characteristics of the saturated nanoliquid porous means are patterns utilizing phenomenological rules and mixing hypotheses. The influence of many parameters about velocity, temperature, and heat transport is found. The highest heat transport is obtained within a simple cylindrical ring contrasted to the rectangle and high circular rings. Increasing the radius from the internal cylinder is to lowers the heat transport. Siddheshwar et al. [15] analytically studied the linear and nonlinear Darcy-Bénard convection about Newtonian nonliquids and Newtonian liquids into cylindrical and cylindrical annuli. They studied the effect of heat transport and the concentric addition 
from a cylinder in the circular cylinder. The twophase representation is utilized within the event of nanoliquids. They noticed that the heat transportation is highest in the event from a cylindrical ring accompanied on such cylindrical and square enclosures. Mebarek et al. [16] numerically studied the convective heat transfer of Titania nanofluids with various basis fluids into a cylindrical ring by a discrete warmth source. A computer code is a developed basis on the finite volume approach coupled with the SIMPLER algorithm. The influences from these parameters on the local Nusselt number are investigated. Mebarek et al. [17] numerically analyzed the effects from the position of a heat fount at the floating convection of nanofluids within an annular system. They examined five various heat fount locations alongside the internal cylinder from the ring. Their goal is to recognize the optimal location from the warmth fount to maximize or minimize heat transportation to many Rayleigh number valors and various volume fractions from the nanoparticle. These results show that the position from the heat fount has a deep influence on the flow motif. The volume fraction from nanoparticles more commands heat transportation into the ring geometry. Lakshmi et al. [18-21] examined analytically the natural convection of nanoliquids into saturated porous cylindrical rings with the inner cylinder under regular mass and heat flow, and the outer cylinder under heat flow. The Darcy pattern and the changed account of the two-phase Buongiorno pattern are utilized. Of the three examples of rings, estimated, shallow rings present the most suitable heat transportation and high rings present the most critical production. The appearance concerning a dilute concentration from nanoparticles significantly improves the warmth transportation within the system. They concluded that a simple cylindrical ring soaked by water and AA7075 lamellar mixture nanoparticles is properly satisfied to heat transfer due to its large efficient thermic conductivity compared to other formed nanoparticles and that a large quadrangular enclosure soaked with water strongly sequences to heat stocking applications. Keerthi et al. $[22,23]$ analyzed the impact of a non-uniform warm state at floating convective transport from nanofluids inside a cylindrical ring formed by two vertical coaxial cylinders. Numerical simulation results show the bicellular flow model to the two non-uniform thermic states across any Rayleigh number ranges. Sankar et al. [24] examined the effects from heat transport conjugated into nanofluids by many nanoparticles so as alumina, titanium oxide, or copper within a closed annular geometry and formed by an internal cylinder having a regular large temperature an outer cylindrical tubing by a fixed below temperature and thermic insulated top and bottom surfaces for a broad Rayleigh number spectrum. These results show that copper-based nanofuid produce more important heat transportation between different nanoparticles. Roy [25] examined the buoyant motion of nanofluids in annular spaces within a rectangular geometry and three inside geometries: elliptical, circular, or rectangular cylinder. He noted that the interior forms have a deep impact on heat transfer flows compared to a square geometry. Sultan et al. [26] studied numerical the heat transfer by natural convection using various nanofluids $\left(\mathrm{Ag}, \mathrm{Cu}\right.$, and $\left.\mathrm{TiO}_{2}\right)$ in a horizontal threedimensional flow and an inclined ring with two heated flippers are fixed on the internal cylinder while the outer cylinder is adiabatic. These numerical effects confirm that while the volume fraction rises, the warmth transfer improved to any valors about the Rayleigh number. The improvement is most important in large Rayleigh numbers.

We conducted a literature search on the associated floatable convection of basic fluids and nanofluids under many geometries. Following detailed analytical and empirical studies, we remarked that the buoyancy movement and heat transfer behavior from nanofluids interior the closed hexagon geometry have not been performed numerically in detail until now to analyze the impacts of several key parameters about the thermal behavior from nanofluids. The work we develop in this manuscript concerns the numerical research from warmth transfer by nanofluids: two coaxial cylinders, a cold external hexagon cylinder, and a heated interior hexagon cylinder. Numerical simulations have been performed to predict the effect of Rayleigh numbers, and the solid volume fraction of nanoparticles, at the flow field and structure, and heat transfer. The current analysis furnishes a maximum amount of details on this subject.

\section{Determining equations to laminar nanofluids}

\subsection{Physical Models}

Figure 1 shows the physical representation from the current work. The cross-section considered consists of an inner hexagon cylinder with six sides, located inside a hexagon cylinder enclosure characterized by (six sides). The surfaces from the outer hexagon fence have been maintained in a fixed lowtemperature $T_{C}$, and the inner hexagon surface is kept in a fixed high-temperature $T_{H}$. During this work, the thermal Rayleigh's number, Rat, ranges from $10^{3}$ to $2.5 \times 10^{5}$. The flow is assumed to be two-dimensional, the flow is convection natural laminar of nanofluid 
( $\mathrm{Cu}-$ water). The nanofluid is considered incompressible and Newtonian by negligible viscous diffusion and pressure working. The thermophysical properties of the nanofluid are assumed constant with the exception of the density, which changes according to the Boussinesq approximation. The Boussinesq approximation is applied to model the buoyancy effect. The acceleration due to gravity acts in the negative $y$-direction.

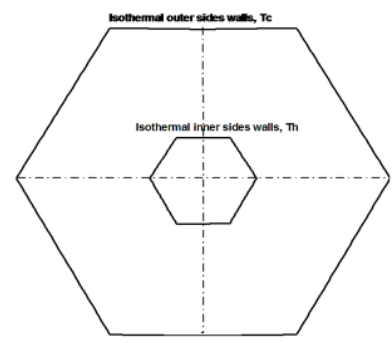

Physical field

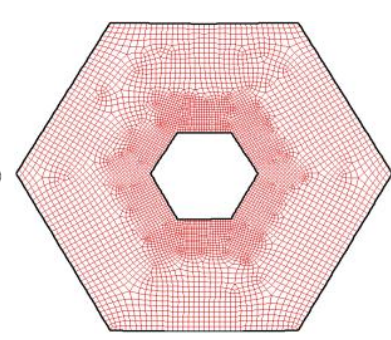

Computational field

\subsection{Mathematical model}

The treatment of the considered physical problem requires the application of governing equations deduced from the classical principles of conservation of mass, momentum, and energy. The determining equations converted into dimensionless models below the non-dimensional variables:

$$
\begin{aligned}
& X=\frac{x}{L} \\
& Y=\frac{y}{L} \\
& U=\frac{u}{\left(\frac{\alpha_{f}}{L}\right)} \\
& V=\frac{v}{\left(\frac{\alpha_{f}}{L}\right)} \\
& \theta=\frac{T-T_{c}}{T_{h}-T_{c}} \\
& P=\frac{p}{\rho\left(\frac{\alpha_{f}}{L}\right)^{2}} \\
& \operatorname{Pr}=\frac{\vartheta_{f}}{\alpha_{f}} \\
& R a_{t}=\frac{g \beta_{f}\left(T_{h}-T_{c}\right) D_{h}^{3}}{\alpha_{f} \vartheta_{f}}
\end{aligned}
$$

By introducing the dimensionless quantities into the conservation equations of mass, motion and energy, we obtain respectively [27-32]:

$$
\begin{aligned}
& \frac{\partial U}{\partial X}+\frac{\partial V}{\partial Y}=0 \\
& U \frac{\partial U}{\partial X}+V \frac{\partial U}{\partial Y}=-\frac{\partial P}{\partial X}+\frac{\mu_{\mathrm{nf}}}{\rho_{\mathrm{nf}} \alpha_{f}}\left(\frac{\partial^{2} U}{\partial X^{2}}+\frac{\partial^{2} U}{\partial Y^{2}}\right) \\
& U \frac{\partial V}{\partial X}+V \frac{\partial V}{\partial Y}=-\frac{\partial P}{\partial Y}+\frac{\mu_{\mathrm{nf}}}{\rho_{\mathrm{nf}} \alpha_{f}}\left(\frac{\partial^{2} V}{\partial X^{2}}+\frac{\partial^{2} V}{\partial Y^{2}}\right)+ \\
& \frac{(\rho \beta)_{\mathrm{nf}}}{\rho_{\mathrm{nf}} \beta_{f}} R a_{t} \operatorname{Pr} \theta
\end{aligned}
$$

$$
U \frac{\partial \theta}{\partial X}+V \frac{\partial \theta}{\partial Y}=\frac{\alpha_{\mathrm{nf}}}{\alpha_{f}}\left(\frac{\partial^{2} \theta}{\partial X^{2}}+\frac{\partial^{2} \theta}{\partial Y^{2}}\right)
$$

\subsection{Estimation from the Nusselt number}

The local Nusselt number adjacent to the outer and interior cylinders and the mean Nusselt number can be estimated as follows [33, 34]:

$$
\begin{aligned}
& N u_{h}=-\frac{k_{\mathrm{nf}}}{k_{f}}\left(\frac{\partial T}{\partial Y}\right)_{Y=0} \\
& N u_{c}=-\frac{k_{\mathrm{nf}}}{k_{f}}\left(\frac{\partial T}{\partial Y}\right)_{Y=1}
\end{aligned}
$$

Table 1 Thermophysical characteristics from the base fluid and $\mathrm{Cu}$ nanoparticles [35]

\begin{tabular}{|l|l|l|l|l|c|}
\hline & $\boldsymbol{\rho}\left(\frac{\boldsymbol{K g}}{\boldsymbol{m}^{3}}\right)$ & $\boldsymbol{C}_{\boldsymbol{p}}\left(\frac{\boldsymbol{J}}{\boldsymbol{k g} \cdot \mathbf{l}} \boldsymbol{K}\left(\frac{\boldsymbol{W}}{\boldsymbol{m} \cdot \boldsymbol{K}}\right.\right.$ & $\boldsymbol{\beta}\left(\frac{\mathbf{1}}{\boldsymbol{K}}\right)$ & $\boldsymbol{\mu ( P s . \boldsymbol { s } )}$ \\
\hline Water & 997.1 & 4179 & 0.613 & $21 \times 10^{-5}$ & 0.000891 \\
\hline Cu & 8933 & 385 & 401 & $1.67 \times 10^{-5}$ & - \\
\hline \multicolumn{5}{|c|}{$\overline{N u}_{\text {avg }}=\frac{1}{D_{h}} \int_{0}^{D_{h}} N u_{x} d x$}
\end{tabular}

\subsection{Nanofluid characteristics}

The thermophysical characteristics of base fluid (water) and nanoparticles (copper) are presented in table 1 . The characteristics of the nanofluids can be get from the properties of the basic fluid and the nanoparticles. The heat capacity and density of the nanofluids are estimated based on the references of Ramiar et al. [36] and Khanafer and Vafai [37].

$$
\begin{aligned}
& (\rho)_{\mathrm{nf}}=\phi \rho_{p}+(1-\phi) \rho_{f} \\
& \left(\rho C_{p}\right)_{\mathrm{nf}}=\phi\left(\rho C_{p}\right)_{p}+(1-\phi)\left(\rho C_{p}\right)_{f}
\end{aligned}
$$

The thermic diffusivity from the nanofluid is

$$
(\alpha)_{\mathrm{nf}}=\frac{K_{\mathrm{nf}}}{\left(\rho C_{p}\right)_{\mathrm{nf}}}
$$

The thermal expansion coefficient of the nanofluid calculated by

$$
(\rho \beta)_{n f}=\phi(\rho \beta)_{p}+(1-\phi)(\rho \beta)_{f}
$$

The efficient dynamic viscosity from the nanofluid determined by Brinkman [38] as

$$
(\mu)_{n f}=\frac{\mu_{f}}{(1-\phi)^{2.5}}
$$

Concerning effective thermic conductivity from the nanofluid, Maxwell [39] presented a model to base density mixing by micron-sized spherical particles, which is

$$
K_{n f}=K_{f} \frac{\left(K_{p}+2 K_{f}\right)-2 \phi\left(K_{f}-K_{p}\right)}{\left(K_{p}+2 K_{f}\right)+\phi\left(K_{f}-K_{p}\right)}
$$

\subsection{Boundary condition}

Solving the system of equations got previously requires the incorporating of boundary conditions to any dependant variable. The temperature conditions are identified on the cylinders. The temperature from the external cylinder is uniform and equal to $T_{c}$. The hot part of the inner cylinder has a constant temperature equal to $T_{h}$. 
These different boundary conditions in dimensional form can be recapitulated as:

The initial conditions are:

$$
\begin{aligned}
& U=V=0 \\
& \theta(X, Y)=0
\end{aligned}
$$

Moreover, the boundary limitations about the problem are:

Inner cylinder (six sides of hexagon)

$$
\begin{aligned}
& U=V=0 \\
& \theta(X, Y)=1
\end{aligned}
$$

External cylinder (six sides of hexagon)

$$
\begin{aligned}
& U=V=0 \\
& \theta(X, Y)=0
\end{aligned}
$$

\subsection{Meshing choice}

The impact of the size and number from nodes at the resolution represented on the warmth transfer to the "heated" active party from the cylinder explained with the average Nusselt number in figure 2. An irregular mesh close to the walls was utilized to resolve more exactly the physical phenoms present in special within the boundary layer zone designated with the presence of large gradients into the parietal regions. To get a mesh-unattached solution, a grill refinement investigation is presented to a hexagon fence by an isothermal internal hexagon at $R a_{t}=10^{4}$ (figure 2). In this paper, eight combinations $(64 \times$ $110,74 \times 130,84 \times 150,94 \times 170,104 \times 190,114 \times$ 210 , and $124 \times 230$ ) of check volumes are utilized to examine the influence of grill size at the precision of foretold results. Figure 2 presents the convergence from the mean Nusselt number, in the heated inner cylinder level by grill refinement. It is remarked that mesh independence is reached by combining control volumes $(114 \times 210)$ in which the mean Nusselt number does not vary importantly with the finer mesh refinement. The accord was determined to be excellent, which corresponds to the current calculation indirectly.

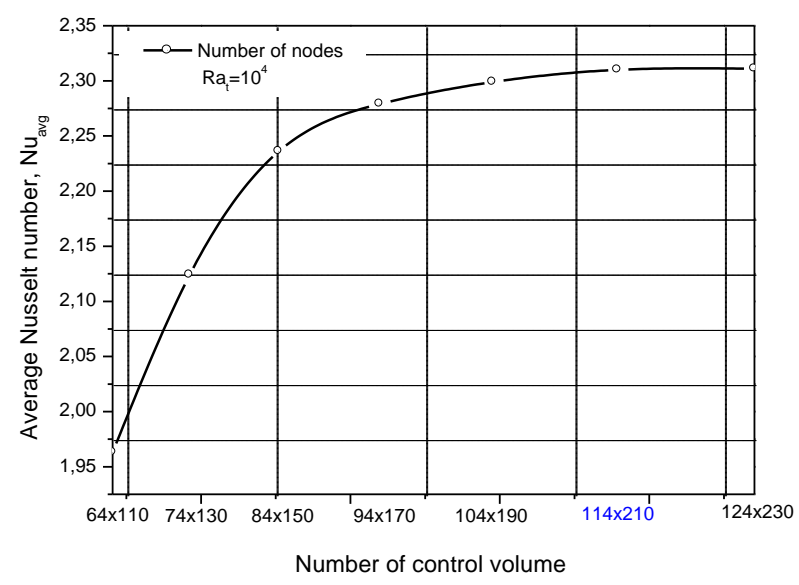

Fig. 2 Convergence from the mean Nusselt number alongside the hot interior cylinder with grill refinement to $R a_{t}=10^{4}$

\section{Validation of results}

In order to verify the accuracy of the numerical results obtained in this work, a validation of the numerical code was made by taking into account certain numerical studies available in the literature. The effect of different Rayleigh numbers on the natural convection of heat transfers in an enclosure delimited by two horizontal confocal elliptical cylinders, filled with air $(\operatorname{Pr}=0.71)$ was studied. Natural convection between horizontal elliptical confocal cylinders by Elshamy [40] was chosen for the validation of the present study. Validations were presented in the form of isotherms and current lines for a different Rayleigh number (fig. 3 (a)). In addition, the local Nusselt number was compared to the reference [40] for different numbers of Rayleigh ranging from $10^{4}$ to $2 \times 10^{5}$ (fig. 3 (b)).

For the case of two confocal horizontal ellipticals, the eccentricity of the inner and outer wall were taken 0.9 and 0.4 , respectively, and the Rayleigh number equal to $10^{4}$ (fig. 3 (a)). The local Nusselt numbers of the inner and outer cylindrical ellipse for two Rayleigh numbers based on the original paper description were plotted in (fig. 3. (b)). The result indicates acceptable agreement with the results presented in Refs [40]. In all cases, the results show that two symmetrical recirculation cells are formed to the right and left of the vertical symmetry of the cavity. This is due to the force of buoyancy produced by temperature gradient.
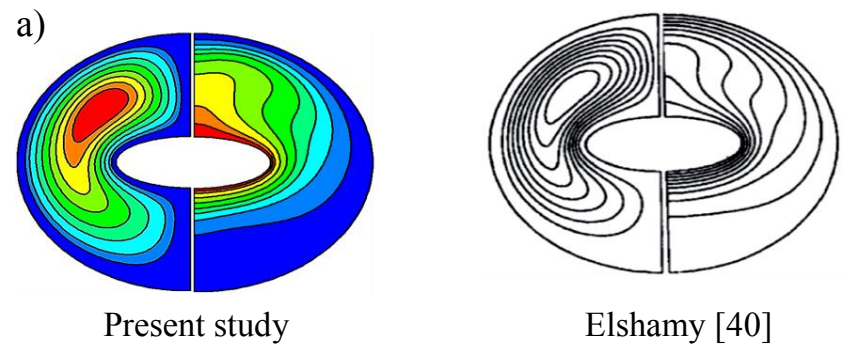

b)

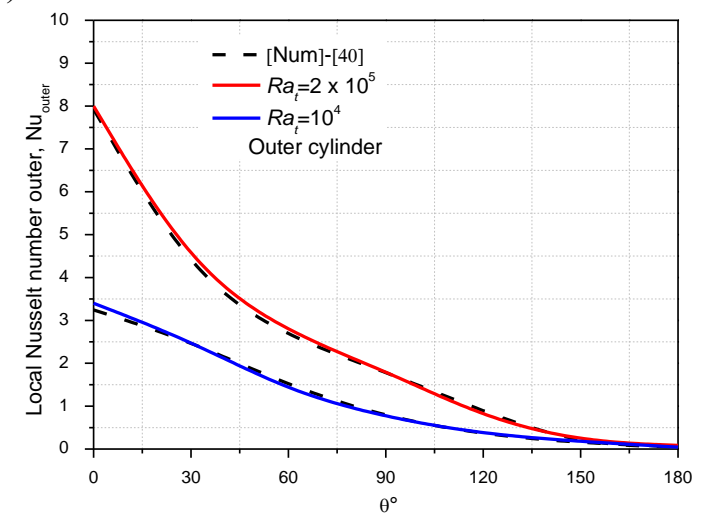




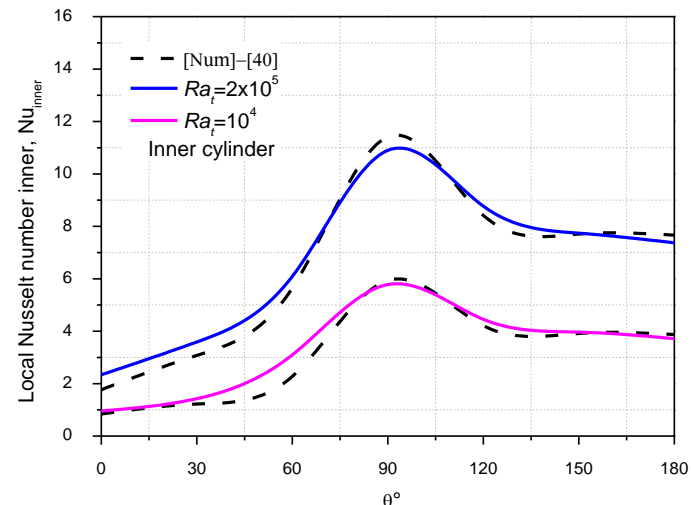

Fig. 3 Validation of natural convection in an elliptical ring. (a) Isocurrent (left half) and isotherms (right half) at $R a_{t}=10^{4}$. (b) Local Nusselt number along inner and outer ellipses and comparison with reference [40].

\section{Results and Analysis}

Within this document, we have offered numerical simulations about natural convection in a closed annular system, this system comprising two confocal cylinders an outer hexagon and an inner hexagon, which controlled at uniform imposed temperatures. The system of equations ruling the problem is resolved numerically utilizing a finite volume approach based on the SIMPLE algorithm. The developed model is first validated from the numerical results. The thermal and dynamic current field is shown. The estimates are carried for thermal Rayleigh numbers of $10^{3}, 10^{4}, 10^{5}$, and $2.5 \times 10^{5}$, and volume fraction of nanoparticles $0 \leq \varnothing \leq 0.12$. We examine the annular space defined by the six sides of the inner hexagon cylinder, and the outer cylinder is a hexagon cylinder designated by (six sides). This annular space is closed which contains a $\mathrm{Cu}$-water nanofluid. The thermophysical characteristics of the copper nanoparticles and the base fluid are presented in table 1 . The external and the internal wall create a temperature gradient.

Figure 4 plotted isocurrent and isotherms for various volume fraction estimations of nanoparticles when the thermic Rayleigh number equals $10^{5}$. It is noted that the isotherms and the streamlines are symmetrical with respect to the fictitious vertical median plane. This figure show that the flow regime is single-cell. On the left side of the plane of symmetry, the flow rotates counterclockwise and at the right lateral, it is within the opposite sens (the particles of the fluid movement upwards below the action from gravity forces along the internal hot wall also then descend to the vicinity of the cold wall of the external cylinder).

At $R a_{t}=10^{5}$, the isotherms grow and ultimately take on the form of a mushroom. The temperature distribution decreases from hot cylinder to cold cylinder. The direction of the deformation of the isotherms corresponds to the path of rotation of the isocurrent. In laminar mode, it can be said that, under the action of the movement of the particles which take off from the hot wall at the level of the axis of symmetry, the isotherms "arch" and move away from the wall at this point. The preferences from the streamlines raise, which confirms that the convection is increasing. In fact, the existence of nanoparticles causes an intensification of isotherms near the hot surface, which indicates an enhancement in the rate of heat transfer.
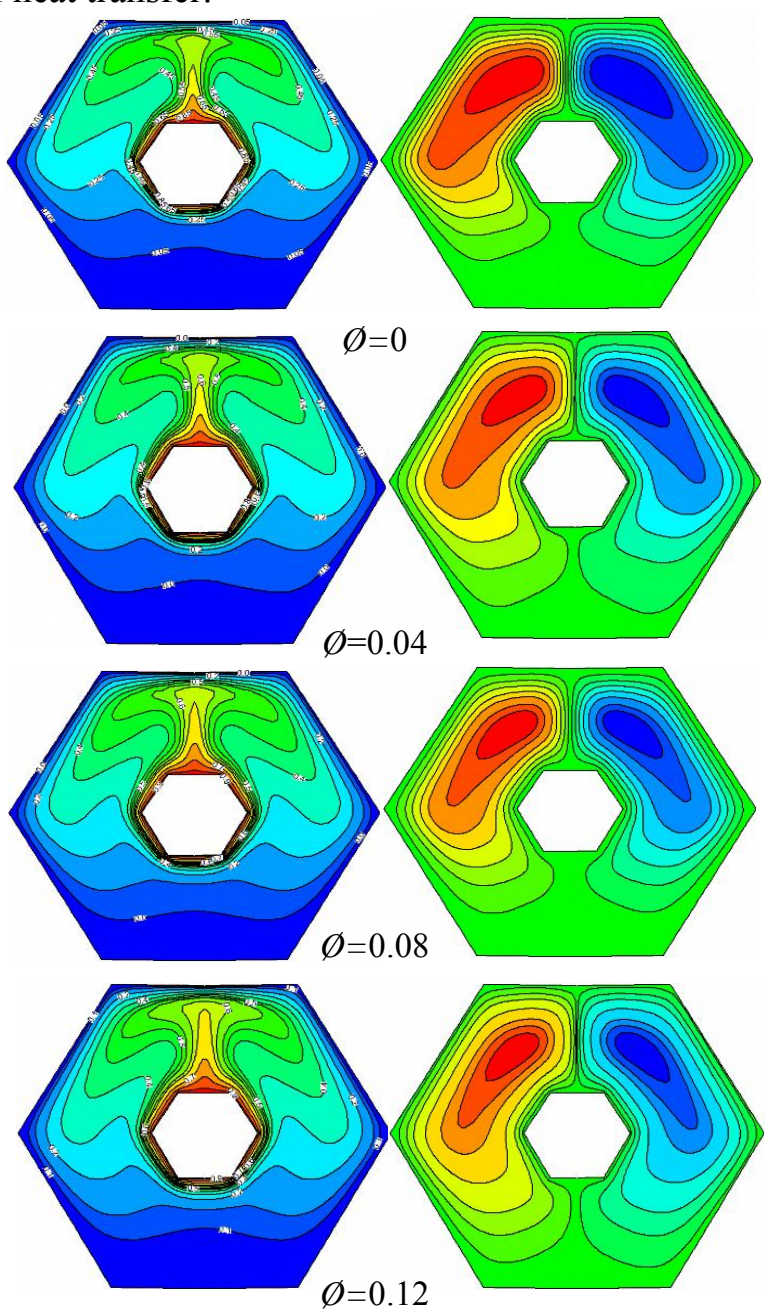

Fig. 4 Isocurrent (right) and Isotherms (left) concerning various values from volume fraction when, $R a_{t}=10^{5}$

Figure 5 presents the contours about the temperature and streamlines when the volume fraction values of the nanofluids are zero $(\varnothing=0)$ for $R a_{t}=10^{3}, 10^{4}, 10^{5}$, and $2.5 \times 10^{5}$. The figure notes that the heated water next to the hot isothermal hexagon (six sides) surface moves upward and then hits the outer hexagon (six sides) cylinder isothermal cold surface. Next striking the cold cylinder, it reverses direction creating a symmetrical rotating vortex. Concerning isotherms, at thermal Rayleigh numbers are between $10^{3}$ and 
$10^{4}$, the isotherms are generally identical, and the heat is transported by conduction. At $R a_{t}\left(=10^{5}\right.$ and $\left.2.5 \times 10^{5}\right)$, the temperature shapes and isocurrent enhance extra confusing, and the vortex intensity raises while the Rayleigh number rises. In that case, the flow highly strikes the ceiling from the exterior cylinder conducts the production of a thin thermic layer. The form of the rotating spirals increases, the isotherms go up, and heat is transferred by convection.
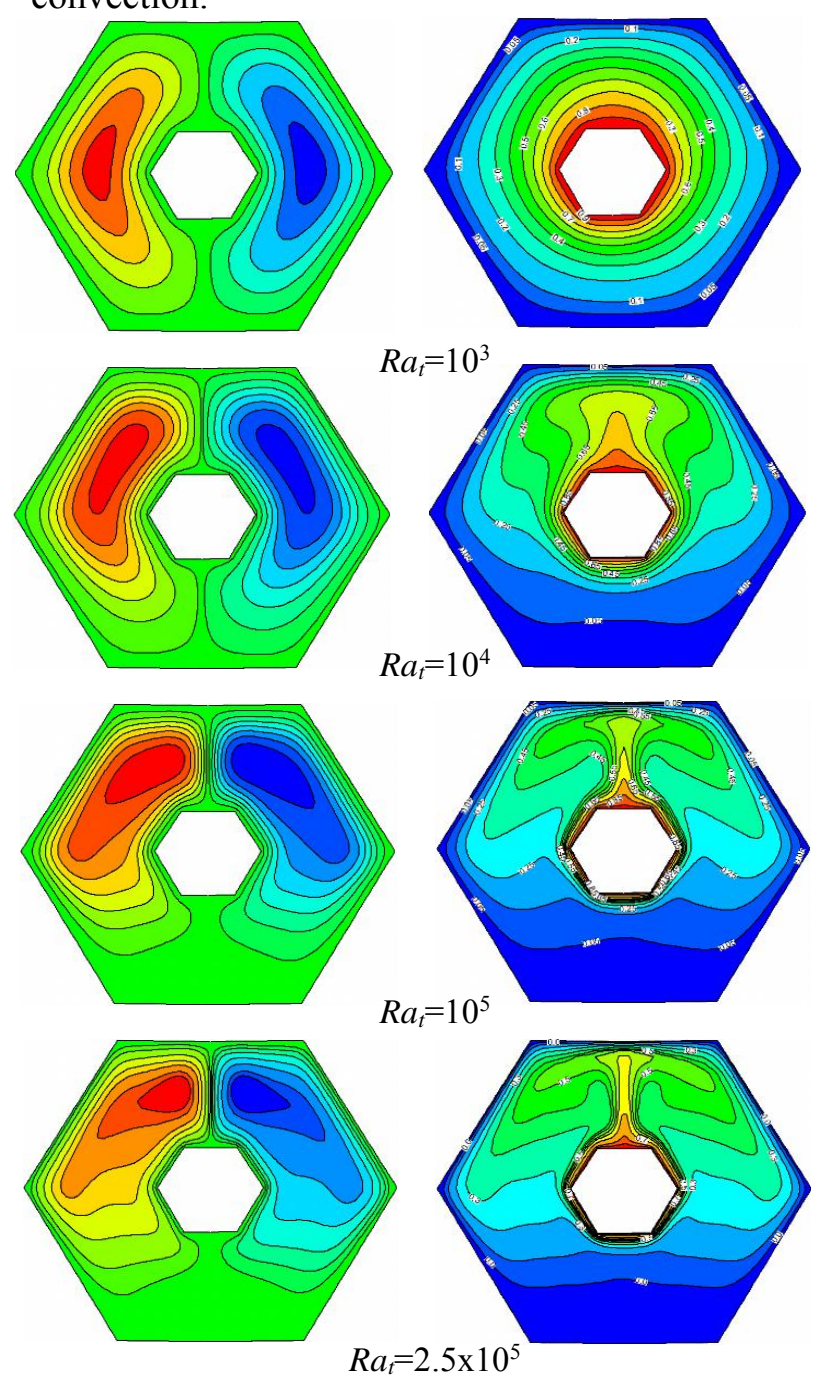

Fig. 5 Isotherms (right) and isocurrents (left) for several values about the thermal Rayleigh number, $R a_{t}$ at $\varnothing=0$

The evolution from the average Nusselt numbers by the nanoparticle volume fraction for various thermal Rayleigh number is presented in figure (6). It is noted that as an adjusted value concerning the thermal Rayleigh number, the mean Nusselt number rises linearly with increasing $\varnothing$. The slope of the line is increased when the thermal Rayleigh number and the volume fraction from the nanoparticles increase, which leads to an increase in the heat flux strongly depending on the thermal Rayleigh number.

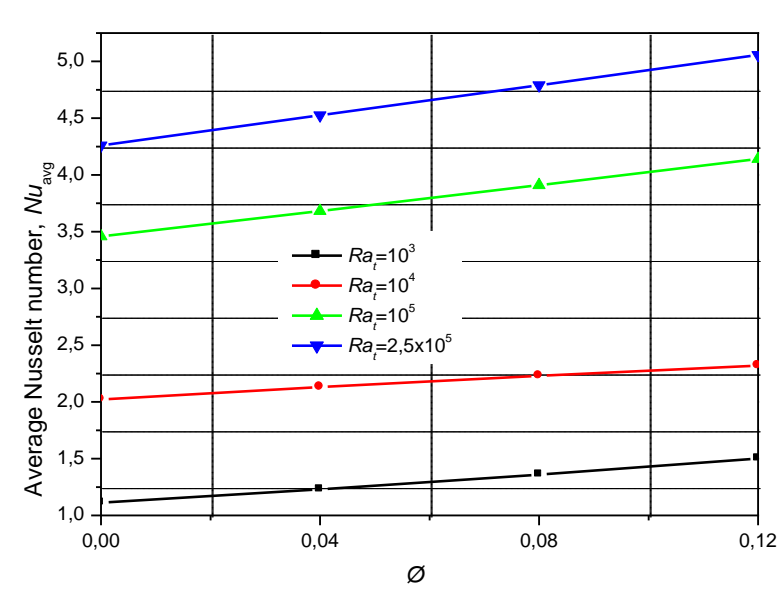

Fig. 6 Evolution of the mean Nusselt number by the nanoparticles volume fraction for various thermal Rayleigh number

Figure. 7 presents the evolution from the mean Nusselt number in the function from the thermal Rayleigh number concerning different values about volume fraction from nanoparticles. It is apparent that with the rise in the volume fraction about nanoparticles, the efficient thermic conductivity from the nanofluid raises, which appears to enhance thermic transportation from the fluid inside the channel and better heat convection.

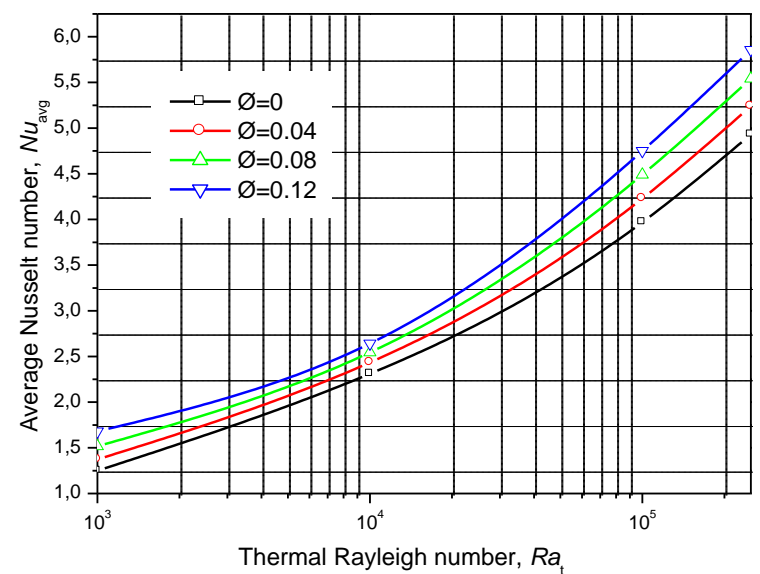

Fig. 7 Evolution from the average Nusselt number round an internal hexagon by the thermal Rayleigh to various values about the nanoparticle volume fraction

The curves of the variation of the local Nusselt numbers with the distance along the walls of the outer and inner polygonal cylinders respectively (i.e., Ao, $\mathrm{Bo}, \mathrm{Co}, \mathrm{Do}$, and $\mathrm{Eo}$ ) and (i.e. ., $\mathrm{Ai}, \mathrm{Bi}, \mathrm{Ci}, \mathrm{Di}$, and $\mathrm{Ei}$ ) for the different volume fraction from thermal Rayleigh number $10^{5}$ nanoparticles are presented in figure (8) and figure (9). A uniform temperature source is applied to the inner polygon sides, which are enclosed in a polygonal enclosure. Changes in local Nusselt numbers are superimposed, and the 
local Nusselt number values for the nanofluid are higher than those for pure water. This is due to the $i$ ncreased conductivity of the nanofluid compared to pure water, which increases heat transfer by diffusion through the inner wall. In the thermal Rayleigh number $10^{5}$, as shown in figure (8), the values of the local Nusselt number increase due to the significant influence of thermal convection and volume fraction values of the nanoparticles. Let us examine figure (8). It starts from the highest value (Ao) in the middle of the upper side of the internal surface (Ai) due to the temperature source effect of the inner cylinder and the presence of small vortices at the internal cylinder and goes down alongside the area of the fence before it approaches the least value near the point Bo. Next, the local Nusselt number gradually rises and reductions until it arrival position Co and Do. Then, the local Nusselt number leaps directly from the area of point Do to point Eo, where the values are almost zero it is the lower part of the enclosure where the fluid is practically stationary.

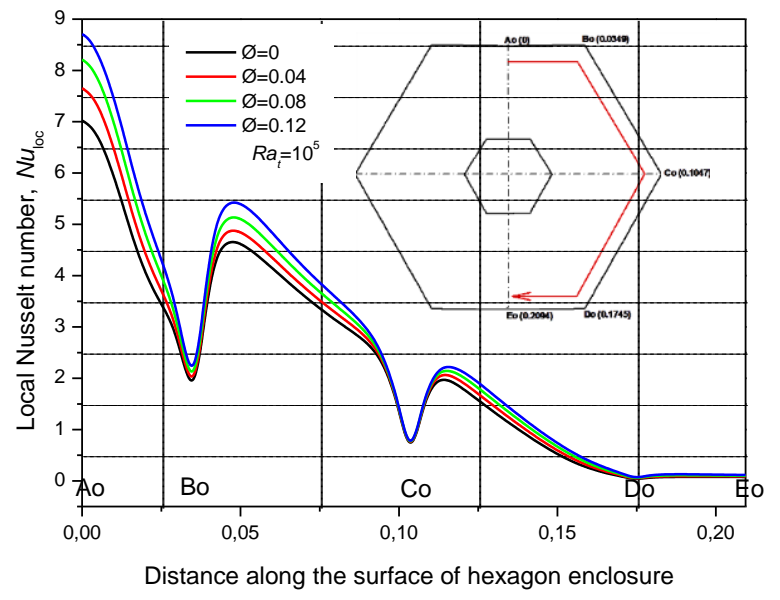

Fig. 8 Evolution of the local Nusselt number alongside the surface (Ao-Bo-Co-Do-Eo) of external the hexagonal enclosure at diverse values from the volume fraction about the nanoparticles for $R a_{t}=10^{5}$.

Figure (9) illustrates the distribution of the local Nusselt number on the internal polygon sides (Ai-Bi, $\mathrm{Bi}-\mathrm{Ci}, \mathrm{Ci}-\mathrm{Di}$, and $\mathrm{Di}-\mathrm{Ei})$. This distribution allowed us to observe that the increase in the volume fraction of the nanoparticles generates an intensification of the natural convection, which implies an increase in the values of the local Nusselt numbers. We also observe in this figure that Nui is maximum in point $\mathrm{Bi}$ and then gradually decreased to a minimum value in the lower part of the internal polygon where the fluid is practically immobile.

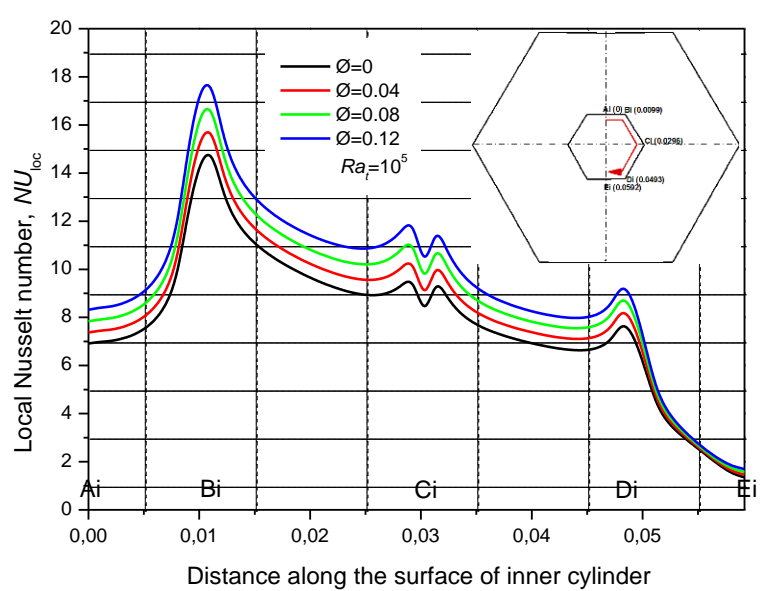

Fig. 9 Evolution of the local Nusselt number alongside the surface (Ai-Bi-Ci-Di-Ei) of internal the hexagonal enclosure at diverse values from the volume fraction about the nanoparticles for $R a_{t}=10^{5}$.

\section{Conclusion}

The enclosures delimited by two cylinders are particularly interesting because they allow a number of configurations and lead to original results, which show in particular that: If the enclosure opposes to the fluid movement sufficiently pronounced constrictions, it is possible to observe multicellular flows even at low values from the thermal Rayleigh number. In this case, the rise concerning the thermal Rayleigh number, i.e. the enhancement of the convection natural, leads to a raise of the vortices.

A numerical investigation from natural convection within a hexagon fence isothermal warmed by an internal hexagon surface with a copper-water-based nanofluid has been performed. The two-dimensional conservation equations of energy, mass, and momentum including the Boussinesq approach were determined utilizing the finite volume technique. The determining parameters are $10^{3} \leq R a_{t} \leq 2.5 \times 10^{5}$, and $0 \leq \varnothing \leq 0.12$ within viewpoint about the obtained results, the conclusions obtained were summarized as follows:

- The contours of the streamlines and the isotherms are symmetrical to the perpendicular line for all situations.

- In high thermal Rayleigh numbers, a thin thermal boundary layer is illustrated at the flow that heavily strikes the ceiling and lower from the outer cylinder.

- The local and mean Nusselt number from a nanofluid are enhanced by enhancing the volume fraction of the nanoparticles.

- To a low thermal Rayleigh number, the conduction process essentially controls the heat transport within the annulus. During the thermal Rayleigh number rises, the role of 
convection becomes dominant. The isotherms grow more confounded and thermal convection is commanding.

- The addition of copper nanoparticles produced an important enhancement within the flow from heat transfer, so the heat transfer rises with the rise in the volume fraction of the nanoparticles.

The research sheds light on this domain and indicates the need for more investigations in the future by examining

- The influence of stationarity and the flow regime.

- An experimental and numerical threedimensional study of this phenom.

- The impacts of the other important control parameters, particularly Prandtl number (Pr)

\section{References:}

[1] Tzeng, S. C, Lin, C. W, Huang, K, Heat transfer enhancement of nanofluids in rotary blade coupling of fourwheel-drive vehicles, Acta Mechanica, Vol. 179, 2005, pp. 11-23.

[2] Leong, J, Lai, F, Natural Convection in a Concentric Annulus with a Porous Sleeve, International Journal of Heat and Mass Transfer, Vol. 49, No. 17-18, 2006, pp 30163027.

[3] Zhou, W, Yan, Y. Y, Xu, J. L, A lattice Boltzmann simulation of enhanced heat transfer of nanofluids, International Communications in Heat and Mass Transfer, Vol. 55, 2014, pp. 113120.

[4] Fayz-Al-Asad, Md, Nur Alam, Md, Ahmad, H, M. M. A. Sarker, M. M. A, M. D. Alsulami, M. D, Khaled, A, Impact of a Closed Space Rectangular Heat Source on Natural Convective Flow through Triangular Cavity, Results in Physics, 2021, Vol. 23, 2021, 104011.

[5] Moghari, R. M, Talebi, F, Rafee, R and Shariat, M, Numerical Study of Pressure Drop and Thermal Characteristics of Al2O3-Water Nanofluid Flow in Horizontal Annuli, Heat Transfer Eng, Vol. 36, No. 2, 2014, pp. 166177.

[6] Izadi, M, Shahmardan, M. M, Behzadmehr A, Richardson Number Ratio Effect on Laminar Mixed Convection of a Nanofluid Flow in an Annulus, International Journal for Computational Methods in Engineering Science and Mechanics, Vol. 14, No. 4, 2013, pp. 304316.

[7] Seyyedi, S, Dayyan, M, Soleimani, S, and Ghasemi, E, Natural Convection Heat Transfer Under Constant Heat Flux Wall in a Nanofluid
Filled Annulus Enclosure, Ain Shams Eng. J, Vol. 6, No. 1, 2015, pp. 267-280.

[8] Dawood, H, Mohammed, H and Munisamy, K, Heat Transfer Augmentation Using Nanofluids in an Elliptic Annulus With Constant Heat Flux Boundary Condition, Case Stud, Therm. Eng, Vol. 4, 2014, pp. 32-41.

[9] Garoosi, F, Jahanshaloo, L, Rashidi, M. M, Badakhsh, A and Ali, M. E, Numerical Simulation of Natural Convection of the Nanofluid in Heat Exchangers Using a Buongiorno Model, Appl. Math. Comput, Vol. 254, 2015, pp. 183-203.

[10] Mehrizi, A. A, Farhadi, M and Shayamehr, S, Natural Convection Flow of $\mathrm{Cu}-$ Water Nanofluid in Horizontal Cylindrical Annuli With Inner Triangular Cylinder Using Lattice Boltzmann Method, Int. Commun. Heat Mass Transfer, Vol. 44, 2013, pp. 147-156.

[11] Arbaban, M, and Salimpour, M, Enhancement of Laminar Natural Convective Heat Transfer in Concentric Annuli With Radial Fins Using Nanofluids, Heat Mass Transfer, Vol. 51, No. 3, 2015, pp. 353-362.

[12] Zoubair Boulahia, Abderrahim Wakif, Ali J. Chamkha, Rachid Sehaqui, 2017, Numerical study of natural and mixed convection in a square cavity filled by a $\mathrm{Cu}$-water nanofluid with circular heating and cooling cylinders, Mechanics and Industry, Vol. 18, No. 5, 2017, pp. 1.

[13] Hamid, M, Khan, Z. H, Khan, W. A, Haq, R. U, Natural convection of water-based carbon nanotubes in a partially heated rectangular finshaped cavity with an inner cylindrical obstacle, Phys. Fluids, Vol. 31, 2019, 103607.

[14] Lakshmi, K. M, Siddheshwar, P. G, Laroze, D, Natural convection of water-copper nanoliquids confined in low-porosity cylindrical annuli, Chin. J. Phys, Vol. 68, No. 4, 2020, pp. 21-136.

[15] Siddheshwar, P. G, Lakshmi, K. M, DarcyBénard convection of Newtonian liquids and Newtonian nanoliquids in cylindrical enclosures and cylindrical annuli, Phys. Fluids, Vol. $31,2019,084102$.

[16] Mebarek-Oudina, F, Convective heat transfer of Titania nanofuids of diferent base fuids in cylindrical annulus with discrete heat source, Heat Transf.-Asian Res, Vol. 48, No. 1,2019, pp. 135-147.

[17] Mebarek-Oudina, F, Keerthi Reddy, N, Sankar, $\mathrm{M}$, Heat source location efects on buoyant convection of nanofuids in an annulus, In Advances in Fluid Dynamics (eds Rushi Kumar, B. et al.), 2020, pp. 923-937 (Springer, 2021). 
[18] Lakshmi, K. M, Laroze, D \& Siddheshwar, P. G, A study of the natural convection of waterAA7075 nanoliquids in low-porosity cylindrical annuli using a local thermal non-equilibrium model, Heat Mass Transf, Vol. 33, 2021, 032018.

[19] Siddheshwar, P. G, Lakshmi K. M, DarcyBénard convection of Newtonian liquids and Newtonian nanoliquids in cylindrical enclosures and cylindrical annuli, Physics of Fluids, Vol. 31, No. 8, 2019, 084102.

[20] Lakshmi, K. M, Siddheshwar, P. G, Laroze, D, Natural convection of water-copper nanoliquids confined in low-porosity cylindrical annuli, Chinese Journal of Physics, Vol. 68, 2020, pp. 121-136

[21] Lakshmi, K. M, Siddheshwar, P. G, Muddamallappa, M. S, Study of rotating Bénard-Brinkman convection of Newtonian liquids and nanoliquids in enclosures, International Journal of Mechanical Sciences, Vol. 188, 2020, 105931.

[22] Keerthi Reddy, N, Sankar, M, Buoyant convective transport of nanofuids in a nonuniformly heated annulus, J. Phys, Conf. Ser, Vol. 1597, 2020, 012055.

[23] Keerthi Reddy, N, Swamy, H. A. K, Sankar, M, Buoyant convective fow of diferent hybrid nanoliquids in a non-uniformly heated annulus, Eur. Phys. J. Spec. Top, Vol. 230, 2021, pp. 1213-1225.

[24] Sankar, M, Reddy, N. K, Do, Y, Conjugate buoyant convective transport of nanofluids in an enclosed annular geometry, Sci Rep, Vol. 11, 2021, 17122.

[25] Roy, N. C, Natural convection of nanofuids in a square enclosure with diferent shapes of inner geometry, Phys. Fluids, Vol. 30, 2018, 113605.

[26] Sultan, K. F, Numerical solution of heat transfer and fow of nanofuids in annulus with fns attached on the inner cylinder, J. Babylon. Univ./Eng. Sci, Vol. 23, No. 2, 2015, pp. 465484.

[27] Sharif M. A. R, Laminar mixed convection in shallow inclined driven cavities with hot moving lid on top and cooled from bottom, Appl. Therm. Eng, Vol. 27,2007, pp. 1036-1042.

[28] M. Shariat, A. Akbarinia, A. Hossein Nezhad, A. Behzadmehr, R. Laur, Numerical study of two phase laminar mixed convection nanofluid in elliptic ducts, Appl. Therm. Eng, vol. 31, 2011, pp. 2348-2359.

[29] M. Izadi, A. Behzadmehr, D. Jalali-Vahida, Numerical study of developing laminar forced convection of a nanofluid in an annulus, Int. J. Therm. Sci, vol. 48,2009, pp. 2119-2129.

[30] R. Lotfi, Y. Saboohi, A. M. Rashidi, Numerical study of forced convective heat transfer of nanofluids: comparison of different approaches, Int. Commun. Heat Mass Transf, vol. 37, 2010, pp. 74-78.

[31] R. Mokhtari Moghari, A. Akbarinia, M. Shariat, F. Talebi, R. Laur, Two phase mixed convection Al2O3ewater nanofluidflow in an annulus, Int. J. Multiph.. Flow, vol. 37, 2011, pp. 585-595.

[32] M. Nuim Labib, Md. J. Nine, H. Afrianto, H. Chung, H. Jeong, Numerical investigation on effect of base fluids and hybrid nanofluid in forced convective heat transfer, Int. J. Therm. Sci, vol. 71, 2013, pp. 163-171.

[33] M. A. R. Sharif, Laminar mixed convection in shallow inclined driven cavities with hot moving lid on top and cooled from bottom, Appl. Therm. Eng, vol. 27, 2007, pp. 1036-1042.

[34] A. Karimipour, M. Afrand, M. M. Bazofti, Periodic mixed convection of a nanofluid in a cavity with top lid sinusoidal motion, Int. J. Mech. Mater. Eng, vol. 1, no. 1, 2010, pp. 3439.

[35] E. Abu-Nada, Z. Masoud, and A. Hijazi, Natural Convection Heat Transfer Enhancement in Horizontal Concentric Annuli Using Nanofluids, Int. Commun. Heat Mass Transfer, vol. 35 , no. 5, 2008, pp. 657-665.

[36] A. Ramiar, A. A. Ranjbar, S. F. Hosseinizadeh, Effect of axial conduction and variable properties on two dimensional conjugate heat transfer of $\mathrm{Al}_{2} \mathrm{O}_{3} \mathrm{eEG} /$ water mixture nanofluid in microchannel, J. Appl. Fluid Mech, vol. 5, no. 3, 2012, pp. 79-87.

[37] K. Khanafer, K. Vafai, A critical synthesis of thermophysical characteristics of nanofluids, Int. J. Heat Mass Transf, vol. 54, 2011, pp. 4410-4428.

[38] H. Brinkman, The Viscosity of Concentrated Suspensions and Solutions, J. Chem. Phys, vol. 20, no. 4, 1952, pp. 571-571.

[39] C. Maxwell, A Treatise on Electricity and Magnetism, Clarendon Press, Oxford, UK. (1881).

[40] M. M. El Shamy, M. N. Ozisik, J. P. Coulter, Correlation for laminar natural convection between confocal horizontal elliptical cylinders, Numer. Heat Transfer, Part A, vol. 18, 1990, pp. 95-112.

\section{Creative Commons Attribution License 4.0} (Attribution 4.0 International, CC BY 4.0)

This article is published under the terms of the Creative Commons Attribution License 4.0

https://creativecommons.org/licenses/by/4.0/deed.en_US 